Melani Wratil

Die Syntax des Imperativs 


\section{studia grammatica}

Herausgegeben von Manfred Bierwisch

unter Mitwirkung von

Hubert Haider, Stuttgart

Paul Kiparsky, Stanford

Angelika Kratzer, Amherst

Jürgen Kunze, Berlin

David Pesetsky, Cambridge (Massachusetts)

Dieter Wunderlich, Düsseldorf 


\section{studia grammatica 62}

Melani Wratil

\section{Die Syntax des Imperativs}

Eine strukturelle Analyse zum Westgermanischen und Romanischen

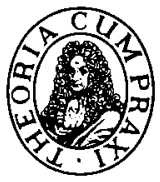

Akademie Verlag 
ISBN 3-05-004189-7

ISSN 0081-6469

(C) Akademie Verlag GmbH, Berlin 2005

Das eingesetzte Papier ist alterungsbeständig nach DIN/ISO 9706.

Alle Rechte, insbesondere die der Übersetzung in andere Sprachen, vorbehalten. Kein Teil des Buches darf ohne Genehmigung des Verlages in irgendeiner Form - durch Photokopie, Mikroverfilmung oder irgendein anderes Verfahren - reproduziert oder in eine von Maschinen, insbesondere von Datenverarbeitungsmaschinen, verwendbare Sprache übertragen oder übersetzt werden.

All rights reserved (including those of translation into another languages). No part of this book may be reproduced in any form - by photoprinting, microfilm, or any other means - nor transmitted or translated into a machine language without written permission from the publishers.

Druck und Bindung: MB Medienhaus Berlin

Printed in the Federal Republic of Germany 


\section{Vorwort}

Die vorliegende Arbeit ist die überarbeitete Fassung meiner Dissertation, die ich im Juli 2004 an der Philosophischen Fakultät der Johann Wolfgang Goethe-Universität in Frankfurt a. M. eingereicht habe.

Allen, die zu ihrer Entstehung beigetragen haben, möchte ich herzlich danken. Mein allererster Dank gilt meinen beiden Betreuern Günther Grewendorf und Peter Gallmann für ihre wohlwollende Förderung und ihr unermüdliches Interesse. Sie haben mir während der vergangenen Jahre stets mit Rat und Tat zur Seite gestanden. Ohne sie wäre diese Arbeit sicherlich niemals fertig gestellt worden.

Bedanken möchte ich mich auch für die wertvolle wissenschaftliche und/oder seelische Unterstützung bei Wolfgang Brauneis, Mark Brüderle, Manfred Consten, Tobias Czybulka, Eva Dobler, Eric Fuß, Jost Gippert, meinen lieben Freunden und Kollegen aus dem Graduiertenkolleg Satzarten: Variation und Interpretation - darunter ganz besonders Magda Schwager und Marina Stoyanova -, Kleanthes Grohmann, Liliane Haegeman, Horst Lohnstein, Rosemarie Lühr, Mark Matter, Cecile Meier, Florian Meier, Peter Öhl, meinen beiden lieben Postdocs Christian Plunze und Malte Zimmermann, Peter Sells, Daniel van den Eijkel, Rainer Voßen, Marina Wratil und Ede Zimmermann.

Abschließend möchte ich Manfred Bierwisch und dem Akademie Verlag für die Aufnahme der Arbeit in der Reihe studia grammatica meinen ganz herzlichen Dank aussprechen.

Köln, Juli 2005 
